FERNANDA AMADEI SAIS

\title{
INFLUÊNCIA DA PREVISIBILIDADE DE EVENTOS NO AMBIENTE E DA INIBIÇÃO MOTORA PROATIVA SOBRE EFEITOS ATENCIONAIS OBSERVADOS EM TAREFAS DE TEMPO DE REAÇÃO
}

Tese apresentada ao Programa de PósGraduação em Fisiologia Humana do Instituto de Ciências Biomédicas da Universidade de São Paulo, para obtenção do Título de Doutor em Ciências.

Área de concentração: Fisiologia Humana

Orientador: Prof. Dr. Marcus Vinícius Chrysóstomo Baldo

Versão original. 


\section{RESUMO}

SAIS, FA. Influência da previsibilidade de eventos no ambiente e da inibição motora proativa sobre efeitos atencionais observados em tarefas de tempo de reação. [Tese (Doutorado em Fisiologia Humana)]. São Paulo: Instituto de Ciências Biomédicas, Universidade de São Paulo; 2017.

A atenção pode ser compreendida como o efeito de mecanismos de tomada de decisão acerca do processamento de estímulos ambientais e da emissão de respostas. Nesse contexto, o aprendizado de regularidades do ambiente e a utilização de predições sobre eventos futuros para alocar o processamento sensorial e implementar estratégias de controle motor são processos determinantes dos efeitos atencionais. Sabe-se que a atenção pode ser orientada para regiões no espaço de acordo com as probabilidades de aparecimento de estímulos alvo em cada local, e que os indivíduos orientam a atenção para o local indicado por uma pista de acordo com a probabilidade de essa pista indicar o lado do estímulo alvo corretamente. Um dos objetivos desse trabalho foi verificar se a atenção pode ser alocada seguindo como pista probabilística a sequência de lados de apresentação dos estímulos alvo gerada por cadeias de Markov probabilísticas de ordem 1 e de ordem 2, que envolvem um aprendizado estatístico implícito mais complexo. Encontramos evidências de que os indivíduos são capazes de alocar a atenção de acordo com esse aprendizado. Além disso, recentemente têm sido discutidas na literatura algumas condições em que se esperaria que uma pista visual tivesse um efeito de benefício atencional sobre os tempos de reação, mas na verdade ela não provoca esse efeito. Essas discussões sugerem que outros efeitos provocados pelo aparecimento de um estímulo, como a inibição motora a processos de mascaramento, poderiam, potencialmente, interagir com efeitos atencionais. Nesse trabalho, nós avaliamos, em dois experimentos com uma condição neutra em que apenas o estímulo alvo é apresentado, o grau de inibição motora proativa implementado durante a realização de uma tarefa e a sua interação com efeitos atencionais. Adicionalmente, utilizamos condições com demarcações e sem demarcações na tela, uma manipulação que poderia alterar a saliência dos estímulos e, portanto, processos de mascaramento e de inibição motora proativa. Resultados com medidas de tempo de reação e de EEG indicam que, nas tarefas utilizadas nesse trabalho, os efeitos da pista visual sobre os tempos de reação podem estar associados a mecanismos de controle motor inibitórios.

Palavras-chave: Atenção. Controle motor. Tomada de decisão. Aprendizado. 


\begin{abstract}
SAIS, FA. Influence of the predictability of events in the environment and of proactive motor inhibition on attentional effects in reaction time tasks. [Ph.D. thesis (Human Physiology)]. São Paulo: Instituto de Ciências Biomédicas, Universidade de São Paulo; 2017.
\end{abstract}

Attention can be understood as the effect of decision making mechanisms determining how to process environmental stimuli and how to respond to these stimuli. As such, the ability to learn regularities in the environment and to use predictions about future events to allocate sensory processing and to implement motor control strategies are strong drivers of attentional effects. It is known that attention can be oriented in space according to the probabilities of stimuli being presented at each location, and that individuals orient attention to the place indicated by a visual cue according to the probabilities of that cue to correctly indicate the location where target stimuli appear. One of our aims in this study was to investigate if attention could be oriented in space using as a probabilistic cue the sequence at which target stimuli would appear at each side of a computer screen, when that sequence could be generated using Markov chains of order 1 and of order 2, which would require complex statistical learning. We found evidence that individuals are able to allocate attention according to this learning. Also, some conditions in which a visual cue that would be expected to cause an effect of benefit on reaction times actually does not have that effect have been recently discussed in the literature. This debates bring about other effects of stimuli appearance, such as proactive motor inhibition and visual masking, that could potentially interact with attentional effects. Here we evaluate the level of proactive motor inhibition in a task and how it interacted with attentional effects, in two experiments using a neutral condition in which only the target stimuli were presented. Additionally, we used conditions with placeholders and without placeholders, a manipulation that could affect stimuli salience and, thus, masking and proactive motor inhibition mechanisms. Results of reaction time measures and EEG indicate that, in tasks such as the ones used in this study, the effects a visual cue has on reaction times can be related to mechanisms of motor inhibitory control. Keywords: Attention. Motor control. Decision making. Learning. 


\section{INTRODUÇÃO}

Ao ler um texto - como esse - você imagina compartilhar com a autora experiências subjetivas associadas à interpretação de palavras e frases, experiências que poderiam ser examinadas introspectivamente e levar, por exemplo, à conclusão sobre a existência de uma faculdade em comum que nos permite seguir o fluxo de ideias do texto e extrair dele significado: a atenção.

Esse é um aspecto fundamental do nosso repertório de experiências cotidianas: quando estamos conscientes temos, sempre, a impressão de estarmos prestando a atenção em alguns locais, objetos, pensamentos, etc., em detrimento de outros.

O início do estudo da atenção foi, de fato, estreitamente relacionado a exercícios de introspecção (James, 2007). Mesmo quando essa área de investigação passou para um estágio centrado em procedimentos empíricos cada vez mais sofisticados, é possível notar que se formou um campo de estudo em que a atenção foi postulada como agente responsável por efeitos que, ainda que implicitamente, têm como origem essas experiências, com resultados interessantes: às vezes parece que temos controle voluntário sobre a orientação da nossa atenção, às vezes parece que a nossa atenção é capturada automaticamente por um estímulo (Näätänen et al., 1980; Awh et al., 2012; Macaluso, Doricchi, 2013); às vezes parece que a nossa atenção é capturada por um estímulo que se destaca no ambiente mas não tem nenhuma relação com o que estamos procurando, às vezes parece que a nossa atenção é capturada por um estímulo parecido com o que estamos procurando (Yantis, 1993; Otten et al., 2016); às vezes parece que a nossa atenção está localizada no ponto para o qual estamos olhando, às vezes parece que ela está em um ponto para o qual não estamos olhando diretamente (Shepherd et al., 1986; Moore et al., 2003); associamos um aumento no grau de atenção (como quando nos concentramos na realização de alguma atividade) à execução de tarefas com maior rapidez e maior acurácia (Prinzmetal et al., 2005) e a um processamento sensorial mais eficiente (Carrasco, Yeshurun, 2009).

O problema com as experiências subjetivas é que elas modulam as perguntas que fazemos - desde "o que vamos jantar hoje?" até "por que o Sol gira em torno da Terra?" - e, muitas vezes, apesar do acúmulo de evidências indicando que pode haver algo errado com essas perguntas, a resistência quanto à adoção de um novo esquema conceitual leva a um atraso na sofisticação do modelo utilizado para interpretar os fenômenos (Khun, 2011). 
Anderson (2011) aborda esse tema, questionando a objetividade e a utilidade do conceito tradicional de atenção para o entendimento dos mecanismos estudados nesse campo de pesquisa - e, mais: mostra como, apesar da importância que esse conceito teve para o desenvolvimento dessa ciência, ele pode, também, gerar questões que são falsos problemas. Os pontos discutidos se devem, em grande parte, ao fato de a atenção ser postulada como a causa de efeitos observados em diferentes condições experimentais e ser concebida de forma vaga como "alguma coisa" que todos saberiam o que é. O autor sugere que, alternativamente, a adoção de uma abordagem dos fenômenos atencionais como processos bayesianos de tomada de decisão tem se mostrado frutífera e poderia ser a linguagem adequada para o estudo dos mecanismos envolvidos (ver Feher da Silva, Baldo (2015) para um exemplo da utilização de modelagem bayesiana para explicar efeitos atencionais).

Nesse sentido, Krauzlis et al. (2014) apresentam evidências neurofisiológicas de que a atenção seria o efeito de mecanismos de tomada de decisão que operam nos núcleos da base envolvendo, principalmente, projeções para o estriado. Os autores sugerem que essas projeções poderiam se originar no colículo superior, passando pelo núcleo medial dorsal do tálamo (que envia sinais diretamente ou indiretamente pelo córtex pré-frontal), pelo núcleo parafascicular (que envia projeções para o núcleo caudado e sinais tanto para a via direta como para a via indireta do estriado), e pela substância negra pars compacta (que envia sinais dopaminérgicos para o estriado). Esse sistema geraria um viés na tomada de decisão, que seria também modulada por sinais provenientes do neocórtex, sendo a atividade moduladora do córtex pré-frontal sobre os núcleos da base inversamente proporcional à previsibilidade dos eventos (Jahanshahi et al., 2015).

Essas ideias sugerem que a compreensão de fenômenos atencionais tradicionalmente estudados na literatura se beneficiaria de um olhar livre das interpretações canônicas dos efeitos observados - e apontam para mecanismos envolvendo os núcleos da base como explicações promissoras para muitos desses efeitos, integrando processos motores, cognitivos e emocionais (Jahanshahi et al., 2015). Para fluência da linguagem e facilitação da compreensão do texto, nesse trabalho quando nos referimos a "efeitos atencionais", "mecanismos de orientação atencional", etc., temos em mente esses mecanismos. 


\subsection{Previsibilidade de uma Pista Visual e Alocação da Atenção}

Os efeitos atencionais estão associados a um comportamento de extrema importância para a sobrevivência do organismo: responder rapidamente e corretamente aos estímulos de interesse. Esse comportamento seria realizado de maneira ideal em um ambiente completamente previsível sem que o organismo precisasse fazer nada além de aprender a associar estímulos específicos a respostas correspondentes. No entanto, o ambiente em que evoluímos e vivemos está longe de ser completamente previsível. Apesar disso, existe alguma previsibilidade, e a habilidade de detectar essas regularidades pode ter sofrido uma pressão evolutiva nos seres humanos para favorecer a busca por padrões (Feher da Silva, Baldo, 2012). Essa ideia é inspirada por muitas evidências de que a busca por padrões é intrínseca ao nosso comportamento e que nós julgamos reconhecê-los mesmo quando eles não estão presentes (Huettel et al., 2002; Oskarsson et al., 2009; Mlodinow, 2011). A estratégia de procurar regularidades no ambiente pode estar associada a mecanismos filogeneticamente antigos e conservados ao longo da história evolutiva, pois pode ser adotada independentemente da consciência do indivíduo (Reber, 1989; Carreiro et al., 2003).

Nesse contexto, a capacidade de um indivíduo monitorar a sua incerteza (metacognição) e detectar quando cometeu um erro pode ser extremamente útil. Há evidências de que o monitoramento de erros associados a predições também pode se dar de maneira inconsciente (van Gaal, Lamme, 2012; Charles et al., 2013; Yin et al., 2016).

A partir da realização de inferências quanto à regularidade do ambiente e do monitoramento de erros associados a essas inferências, o organismo toma uma decisão que pode se dar de maneira inconsciente e automatizada - sobre como lidar com os estímulos ambientais. Essa decisão dá origem aos diferentes efeitos atencionais e, em última análise, resulta em uma resposta motora. Uma questão importante nesse processo é: onde, e quando, vai aparecer um estímulo que pode exigir uma resposta?

Essa é a situação que um indivíduo encontra à sua frente quando participa de um experimento de psicofísica, em que deve responder o mais rápido e/ou corretamente possível a um estímulo que será apresentado na tela de um computador. Sabe-se que os seres humanos são capazes de utilizar a estrutura probabilística do ambiente a partir da frequência de aparecimento de um estímulo em uma região do espaço para selecionar os 
locais com maior probabilidade de apresentação de um estímulo para um processamento sensorial intensificado (Ciaramitaro et al., 2000). Efeitos atencionais são observados em benefícios nos tempos de reação e na acurácia da resposta a partir do aprendizado de contingências probabilísticas complexas (Druker, Anderson, 2010). Esse aprendizado se dá de maneira implícita e sem nenhuma outra indicação a respeito do aparecimento dos estímulos além da frequência com que eles aparecem em cada região.

Em muitos experimentos, são apresentadas pistas que indicam corretamente o local de aparecimento do estímulo alvo na maioria das vezes (ver, por exemplo, Posner, Cohen (1984) e Vossel et al. (2006)). A tomada de decisão do indivíduo sobre que local do espaço priorizar para processamento parece envolver dois sistemas, um de natureza mais cognitiva e explícita, e outro que funciona de maneira mais automática, implícita.

Quando o indivíduo é informado sobre a previsibilidade da pista, tudo que ele precisaria fazer para obter os melhores resultados possíveis é seguir a instrução de orientar, sempre, a atenção para o local indicado pela pista, pois orientar a atenção para o local previsto pela pista os levaria a ter um benefício todas as vezes em que o alvo aparecesse naquele local. Há evidências, no entanto, de que não é essa a estratégia adotada. Os indivíduos parecem adotar uma estratégia de pareamento de probabilidades (Jonides, 1980, 1983; van der Haijden, 1989), orientando a sua atenção para um lado da tela com a mesma frequência que a pista indica aquele lado, o que diminui as suas chances de acertar o lado de aparecimento do alvo. Esses resultados foram obtidos tanto para pistas centrais como para pistas periféricas, preditivas ou não preditivas.

A relação entre escolhas explícitas e a orientação atencional foi estudada de maneira mais direta em um trabalho de Geng et al. (2013), em que os voluntários deveriam indicar, no início da tentativa, em que lado acreditavam que apareceria o alvo e, após o aparecimento do alvo, deveriam apertar um botão o mais rapidamente possível. O alvo aparecia com probabilidade de 0,7 em um lado da tela e 0,3 do outro - os voluntários não foram informados sobre essa relação e, para as escolhas explícitas, utilizaram a estratégia de pareamento de probabilidades, escolhendo o lado em que o alvo aparecia com probabilidade 0,7 em $70 \%$ das vezes e o lado em que o alvo aparecia com probabilidade 0,3 em $30 \%$ das vezes. Os tempos de reação também variaram de acordo com o esperado, com efeitos atencionais indicados por tempos de reação menores para o lado de maior probabilidade de aparecimento do alvo do que para o lado de menor probabilidade. Quanto 
à relação entre as escolhas explícitas e a orientação atencional, os autores concluem que a atenção é orientada de acordo com a escolha explícita apenas no caso das escolhas mais prováveis; no caso das escolhas menos prováveis, os voluntários teriam adotado uma estratégia diferente para a orientação atencional e, por isso, não teria sido observado um efeito significativo da escolha explícita sobre os tempos de reação no caso dessas escolhas.

É possível que, quando o voluntário faz pareamento de probabilidades, ele saiba, implicitamente, que está acertando as probabilidades de aparecimento dos estímulos e seu sistema cognitivo tende a persistir na busca por padrões em vez de desistir e maximizar sua chance de acerto (Gaissmaier; Schooler, 2008; Feher da Silva, 2011; Victorino, 2012). Nesse caso, como há tempo para que o indivíduo possa deliberar, ele estaria priorizando alocar a sua atenção para o local que escolhe mais vezes devido a uma maior modulação atencional descendente ativada pela previsão de que seria mais provável acertar nessa escolha e, nas escolhas de menor frequência, a maior incerteza do indivíduo quanto à sua própria escolha o levaria a modular a orientação atencional de modo a não priorizar o processamento relacionado a essa escolha em uma situação em que é mais provável que esteja errado. Quando se administra modafinil aos participantes, esse padrão é revertido e a alocação atencional segue tanto a escolha explícita mais provável como a menos provável - o modafinil é um inibidor seletivo da recaptação de dopamina que atua principalmente no estriado; o aumento na disponibilidade de dopamina diminuiria a aversão ao risco (Tom et al., 2007).

No caso da alocação automática da atenção, não há tempo para uma tomada de decisão que se baseie no sistema deliberativo, de modo que essa decisão deve ocorrer por um aprendizado procedural que integra informações sobre os resultados de tentativas anteriores e/ou por estratégias locais baseadas no resultado da resposta mais recente, como, por exemplo, repetir a resposta caso tenha acertado ou mudar a resposta caso tenha errado (Otto et al., 2011; van der Meer et al., 2012). Ambas as estratégias poderiam dar origem aos resultados que indicam um pareamento de probabilidades na alocação atencional, mas apenas no primeiro caso esperar-se-ia que a alocação atencional seguisse o aprendizado de um padrão subjacente às probabilidades de aparecimento do alvo em uma situação em que os incentivos para repetir a escolha ou mudar a escolha fossem equivalentes. 


\subsection{Efeitos da Apresentação de uma Pista sobre o Controle da Inibição Motora}

As predições a respeito de eventos ambientais e a posterior alocação atencional a partir de decisões tomadas de acordo com essas predições estão necessariamente vinculadas a uma ação, que pode ser emitir uma entre duas ou mais respostas possíveis inclusive não executar nenhum movimento.

Os mecanismos de controle motor são caracterizados fundamentalmente por processos de controle inibitório regulados por conexões entre os núcleos da base e regiões frontais do córtex. O controle inibitório proativo está associado a comportamentos intencionais e é implementado antecipadamente de modo a evitar uma resposta indesejável a um estímulo previsto antes que ele apareça; o controle inibitório reativo ocorre após o aparecimento de um estímulo que desencadeie a ativação automática de mecanismos sensório-motores que levariam a uma resposta indesejável e pode se tornar automático com o aprendizado (Braver, 2012; Stuphom, Emeric, 2012; Jahanshahi et al., 2015; Stuphorn, 2015).

Imagina-se que o modo padrão de funcionamento do sistema nervoso seria, ao menos em parte, um estado de inibição proativa tônica de mecanismos de iniciação de movimento, que seria liberado temporariamente pelo sistema executivo de acordo com a previsibilidade do ambiente e o risco de que uma ativação motora automática desencadeasse uma resposta inapropriada (Jaffard et al., 2008; Criaud et al., 2012). Desse modo, uma maior incerteza do ambiente tende a evitar a transição desse estado para um estado automático de processamento sensório-motor; e também a previsão de aparecimento de um estímulo capaz de ativar uma resposta motora indesejada, que leva à estratégia de manter o estado de inibição motora proativa.

O córtex pré-frontal dorso-medial pode ser responsável pelo controle da inibição proativa (Jaffard et al., 2008; Stuphorn, Emeric, 2012) e atuar por meio da supressão de mecanismos neurais envolvidos no comando de iniciação motora (Jaffard et al., 2008; Criaud et al., 2012). Além disso, o córtex pré-frontal dorso-medial também enviaria sinais que modulariam a atividade motora a partir de um mapa com valores associados à recompensa das ações (executar ou inibir uma resposta). Cada ação teria um peso variável de acordo com o histórico de recompensa, levando a diferentes estados de motivação. O sinal resultante 
controlaria a excitabilidade do sistema motor e, portanto, também a relação entre a velocidade e a acurácia da resposta (Stuphorn, Emeric, 2012).

O limiar de resposta para esse tipo de controle seria implementado gradualmente pela modulação da ativação do córtex para o estriado, envolvendo ciclos via indireta-estriado, com o núcleo subtalâmico exercendo um papel importante para liberar o sistema motor do controle inibitório. O papel do núcleo subtalâmico também é considerado importante nos mecanismos de inibição reativa, por meio da via hiperdireta, que o conecta diretamente ao córtex, e pode agir como uma interface direta entre o sistema executivo e o sistema motor (Favre et al., 2013). Mecanismos de inibição proativa e de inibição reativa podem atuar em conjunto para garantir que as respostas aos estímulos sejam adequadas (Meyer, Bucci, 2016).

Tradicionalmente se considera que a apresentação de uma pista periférica antes do aparecimento de um estímulo alvo tem efeitos facilitadores sobre a resposta de detecção do alvo. Recentemente, no entanto, foi demonstrado que esses efeitos não se sustentam quando é utilizada como controle uma condição em que a inibição proativa é minimizada (Jaffard et al., 2007; Boulinguez et al., 2008, 2009; Albares et al., 2011). Nesses trabalhos, o efeito da pista foi comparado a uma condição controle em que apenas o alvo é apresentado durante um bloco de tentativas (bloco puro), em vez de a condição controle e a condição com pista serem apresentadas no mesmo bloco (bloco misto), como normalmente ocorre nesse tipo de experimento. Com essa comparação, descobriu-se que, na verdade, a pista não exerce um efeito facilitador sobre a resposta ao alvo nessas tarefas - pelo contrário, ela aumenta o grau de inibição proativa, observada a partir dos tempos de reação mais altos para a condição controle no bloco misto do que no bloco puro.

O grau de inibição proativa implementado durante a realização da tarefa aumenta tanto porque a pista evoca uma ativação motora automática que precisa ser evitada, como porque ela diminui a previsibilidade quanto ao aparecimento do próximo estímulo. Nesse caso, o aparecimento da pista teria o efeito de liberar essa inibição proativa. Quando o alvo aparece em um período curto após a apresentação da pista, a inibição proativa ainda não teria sido suficientemente diminuída e, por isso, o tempo de reação seria maior do que o observado em uma condição controle (apenas alvos apresentados em blocos puros). Quando apenas o alvo é apresentado em um bloco misto, a inibição proativa seria máxima e só seria liberada após o aparecimento do alvo (Boulinguez et al., 2008). 
Um estímulo mais saliente pode evocar uma maior ativação sensório-motora automática, que envolveria uma inibição reativa para evitar uma resposta inadequada. Desse modo, a saliência dos estímulos altera a representação que os indivíduos têm da tarefa, modulando o controle da inibição proativa de maneira que a ativação sensóriomotora seja previamente inibida. Os tempos de reação para o alvo são mais baixos quando a pista tem saliência baixa e mais altos quando a pista tem saliência alta, mas a relação entre a saliência da pista e a saliência do alvo parece ser o fator mais importante: quando a pista e o alvo têm saliência equivalente o grau de inibição motora é equivalente, quando a pista é menos saliente que o alvo a inibição motora é menor, e quando a pista é mais saliente que o alvo a inibição motora é maior (Wardak et al., 2012).

Esses resultados mostram a importância do uso de uma condição controle apropriada para compreender os efeitos observados em um experimento. Os efeitos de custo associados à orientação espacial por uma pista visual, por exemplo, são mais consistentes do que os efeitos de benefício, mas, em muitos experimentos, utiliza-se apenas a comparação entre uma condição válida e uma condição inválida (Albares et al, 2011).

Em tarefas de tempo de reação de escolha, a existência de mais de uma resposta possível leva à competição entre elas, que poderia ser implementada por meio de mecanismos inibitórios: a ativação de estruturas motoras envolvidas na resposta necessária está associada à inibição de estruturas envolvidas na resposta alternativa (Burle et al., 2004). Os processos de inibição proativa que interagem com os efeitos da pista em tarefas de detecção possivelmente afetam também tarefas de escolha, e muitos resultados obtidos nesses experimentos poderiam ser reinterpretados a partir dessa observação (Jaffard et al., 2007). Uma evidência nesse sentido é o achado de que o controle da inibição proativa pode agir de maneira seletiva sobre a representação motora de uma resposta de acordo com os objetivos envolvidos na tarefa (Cai et al., 2011). É possível que esse controle possa agir de maneira inespecífica quando a tarefa exige a identificação do estímulo alvo antes da emissão de uma resposta.

Uma linha de pesquisa com resultados interessantes na literatura, mas em que não é comum discutir-se o controle da inibição proativa, propõe um modelo de captura contingente da atenção, de acordo com o qual uma pista irrelevante capturaria a atenção apenas se tivesse características correspondentes às configurações atencionais da tarefa. Esse modelo foi inicialmente proposto por Folk et al. (1992), a partir de resultados de uma 
tarefa de escolha em que foram utilizados pistas e alvos que poderiam ser ou um estímulo de aparecimento abrupto, ou um estímulo que se destacasse pela cor, e essas características foram manipuladas ortogonalmente, de modo que todas as combinações foram apresentadas. Quando a pista e o alvo possuíam a mesma característica, a pista capturou a atenção, quando a pista e o alvo tinham características diferentes, a pista não capturou a atenção.

A explicação dos autores para esses resultados é que as configurações atencionais seriam ajustadas de acordo com as demandas de uma tarefa, de tal modo que estímulos que tivessem características compatíveis com as configurações atencionais implementadas capturariam a atenção de maneira involuntária, mesmo que isso representasse um custo para o desempenho do indivíduo. Por outro lado, quando o estímulo não tivesse características compatíveis com essas configurações, essa captura atencional involuntária poderia ser evitada. Essa interação entre a contingência dos estímulos e os efeitos sobre os tempos de reação observados foi bastante replicada na literatura (ver Burnham, 2007 para uma revisão), e as discussões em torno desse modelo são um excelente exemplo dos problemas apontados por Anderson (2011), já discutidos aqui. Há um debate na literatura, que já foi mais dicotômico no passado, entre pesquisadores que defendem esse modelo e pesquisadores que defendem modelos em que essa modulação não consegue evitar a captura da atenção por um estímulo saliente (Folk et al., 1994; Remington et al., 2001; Burhnam, Neely, 2008; Folk et al., 2009; Theeuwes, 2010; Awh et al., 2012; Carmel, Lamy, 2015).

O que nos interessa nesse trabalho é que houve uma mudança na previsibilidade do ambiente entre o contexto dos experimentos 1 e 2 e o contexto do experimento 3 no estudo original de Folk et al. (1992), descrito a seguir. Como já vimos, a previsibilidade do ambiente está associada ao controle da inibição proativa, de modo que um ambiente com maior previsibilidade leva a uma menor inibição proativa.

Nos dois primeiros experimentos, cada condição foi apresentada de maneira blocada, e o indivíduo tinha condições de prever os eventos em $100 \%$ das tentativas, o que minimizaria a inibição proativa associada à realização da tarefa. No terceiro experimento, a pista era apresentada em todas as tentativas, mas não tinha nenhuma relação com o local de aparecimento do estímulo alvo - nesse caso, a pista adiciona um fator de incerteza à tarefa, o que levaria a uma maior inibição proativa. Os valores de tempo de reação obtidos 
na condição em que tanto a pista como o alvo eram estímulos de aparecimento abrupto são compatíveis com essa hipótese. No experimento em que a pista era $100 \%$ válida ou $100 \%$ inválida em cada bloco em relação ao local de aparecimento do alvo, o tempo de reação médio, considerando as condições válida e inválida, foi 497 ms; no experimento em que a pista não era preditiva, foi de aproximadamente $587 \mathrm{~ms}$ (os autores não fornecem os números exatos nesse segundo caso, mas o valor é razoavelmente acurado observando-se os gráficos). Um ponto relevante é que essa mudança nos tempos de reação médios foi acompanhada por uma alteração na magnitude dos efeitos atencionais: na condição $100 \%$ preditiva, esse efeito foi de $62 \mathrm{~ms}$ e, na condição não preditiva, de $22 \mathrm{~ms}$. É possível que, caso a previsibilidade da pista diminuísse ainda mais, como, por exemplo, pela adição de tentativas em que a pista não fosse apresentada e/ou pela adição de tentativas em que o alvo não fosse apresentado após a pista, o efeito atencional deixasse de ser observado, ainda que a pista tivesse uma característica relevante para a realização da tarefa.

Esses resultados indicam a possibilidade de que haja uma modulação da inibição proativa associada ao aparecimento de uma pista de orientação espacial em uma situação em que a tarefa consiste em identificar o alvo e, ainda, que o controle da inibição proativa possa interagir com os efeitos atencionais.

Isso poderia explicar resultados de experimentos em que tanto a pista como o alvo eram estímulos de aparecimento abrupto mas não foram observados efeitos atencionais significativos. Esse é o caso de estudos em que o uso de demarcações na tela no local de aparecimento dos estímulos, frequentemente utilizadas em experimentos atencionais inspirados nos procedimentos de Posner e Cohen (1984), parecem interagir com os efeitos atencionais, de maneira que estes são mais robustos quando são utilizadas demarcações do que quando elas não são utilizadas (Fuga, 2002; Guedes, 2007; Sais, Ribeirodo-Vale, 2013; Gonçalves, 2015). É possível que essa interação ocorra porque a atenuação do processamento da pista e do alvo pela presença das demarcações ao seu redor (Stemmler et al., 1995; Angelucci, Bullier, 2003) não ocorre quando não há demarcações. Essa mudança poderia alterar a saliência da pista e do alvo que, como vimos, podem interagir com a inibição proativa.

No entanto, mecanismos de mascaramento também poderiam explicar esses efeitos. Estímulos ao redor da apresentação de um alvo são conhecidos por causar mascaramento por paracontraste, e os efeitos facilitatórios e inibitórios do estímulo 
mascarador sobre a percepção do alvo interagem de acordo com a polaridade do contraste, a assincronia entre o início dos estímulos, e variam de acordo com a separação entre o alvo e o estímulo mascarador (Kaitz et al., 1985; Macknik, Livingstone, 1998; Macknik et al., 2000; Keysers, Perret, 2002; Kafaligönül et al., 2009). Essa variedade de efeitos de mascaramento poderia explicar alguns dos efeitos considerados atencionais, ou interagir com efeitos atencionais afetando o resultado final sobre o tempo de reação (Gonçalves, 2015).

Além disso, é possível que as demarcações sejam importantes para o aparecimento do efeito atencional em algumas condições porque a atenção seria mais facilmente orientada para as demarcações, processadas como objetos presentes na cena antes do aparecimento do alvo, do que para um local do espaço. De fato, o componente $\mathrm{N} 2 \mathrm{pc}$ do potencial relacionado a eventos do EEG, interpretado na literatura como um marcador para o efeito atencional (Hopf et al., 2000; Eimer, Kiss, 2008; Kiss et al., 2008; Eimer et al., 2009; Mazza et al., 2009; Eimer, Kiss, 2010), foi observado antes do aparecimento do alvo quando as demarcações estavam na tela, mas não quando as demarcações não estavam presentes (Woodman et al., 2009). Mesmo quando é utilizada uma pista preditiva em relação ao local em que o alvo será apresentado e não preditiva quanto ao objeto em que o alvo será apresentado, os indivíduos priorizam o processamento do objeto indicado pela pista (He et al., 2004).

Assim, parece haver pelo menos três fatores associados à apresentação de uma pista visual periférica que poderiam interagir com o aparecimento de efeitos atencionais em tarefas de tempo de reação: 1) a previsibilidade da pista, que poderia influenciar o controle inibitório do sistema motor; 2) saliência da pista e saliência do alvo, que poderia tanto influenciar o controle inibitório do sistema motor como mecanismos de mascaramento; e 3) mecanismos de focalização do processamento sensorial que priorizem objetos, em vez de locais, em uma cena visual.

\subsection{Análises Comportamentais}

\subsubsection{Cronometria mental}

A utilização de tempos de reação como medida para inferir processos mentais não observáveis durante a realização de uma tarefa é utilizada desde o final do século XIX, com o desenvolvimento do método de subtração de Donders e do método dos fatores 
aditivos de Sternberg - e, até hoje, é importante para a compreensão de processos cognitivos, em conjunto com novas tecnologias como, por exemplo, potencial relacionado a eventos do eletroencefalograma, ressonância magnética funcional, e tomografia por emissão de pósitrons (Posner, 2005).

Em geral, tempos de reação para tarefas de escolha são aproximadamente 100 ms mais lentos do que tempos de reação para tarefas de detecção, e, quando se considera que diferenças nos tempos de reação se devem à adição de processos ou à influência em uma das etapas envolvidas em processamentos sequenciais, propõe-se que essa diferença poderia corresponder ao tempo necessário para distinguir entre os estímulos possíveis ou, em grande parte, para selecionar uma resposta (Luce, 1986). Esse tipo de informação pode ser relevante não apenas para se considerar a adição de uma etapa de processamento (identificação dos estímulos e/ou seleção da resposta, nesse caso) mas, de maneira importante, para identificar mudanças na representação que o indivíduo tem da tarefa que possam alterar a sua estratégia - como, por exemplo, a implementação de uma maior inibição proativa para evitar o início de respostas motoras antes que o estímulo alvo seja identificado.

A análise dos tempos de reação utilizando a média como parâmetro é um método padrão em muitas linhas de pesquisa da psicofísica. Um problema com esse método é que a curva de distribuição dos tempos de reação não é uma gaussiana; ela é melhor modelada como uma ex-Gaussiana, a mistura de uma curva gaussiana com uma exponencial. Essa distribuição é assimétrica, com a parte positiva da distribuição alongada. Assim, os efeitos observados nas médias podem ser produzidos tanto por um deslocamento na distribuição dos tempos de reação, como por um alongamento maior na parte exponencial da curva, que corresponde aos tempos de reação mais lentos (Balota, Yap, 2011).

Utilizar um parâmetro de medida central como a média quando a diferença entre duas condições pode estar em outro parâmetro da curva reduz o poder de um teste de hipótese como, por exemplo, a análise de variância para medidas repetidas, que é muito utilizada na literatura sobre efeitos atencionais.

Para contornar esse problema, diversas alternativas podem ser consideradas (Ratcliff, 1993; Whelan, 2008). Talvez uma das mais comuns seja calcular a mediana dos tempos de reação em vez de calcular a média, porque ela é menos suscetível à influência dos valores extremos. Outra possibilidade é fazer uma transformação nos dados, como utilizar o 
inverso dos tempos de reação (média harmônica), o que pode tornar a distribuição mais próxima da gaussiana (Osborne, 2002).

Tanto no caso de se usar a mediana, como no caso de se fazer a transformação inversa, a probabilidade de cometer erro do tipo I parece não ser afetada. A transformação inversa tem um poder maior na maioria dos casos quando comparada com outros métodos, mas quando a variabilidade entre os indivíduos é alta o uso da mediana pode ter um poder maior do que a transformação inversa (Ratcliff, 1993). A mediana, no entanto, deve ser utilizada com cuidado, pois ela pode ser superestimada; quanto mais enviesada for a parte positiva da curva e menor o tamanho da amostra, mais superestimada será a mediana. 0 uso da mediana é particularmente problemático quando as condições de um experimento não têm o mesmo número de tentativas (Miller, 1998).

Nesse trabalho nós utilizamos, num primeiro momento, a mediana dos tempos de reação e a média do inverso dos tempos de reação (a que nos referimos aqui como velocidade de reação), fazendo uma breve comparação entre os efeitos obtidos com as duas variáveis. Para as análises post hoc e discussão dos resultados, optamos por utilizar as análises feitas com a transformação inversa, pois em algumas das nossas condições experimentais o número de tentativas é relativamente pequeno, e nem sempre temos o mesmo número de tentativas em todas as condições (tanto pelo desenho experimental, como por exclusão de tentativas incorretas da análise em casos em que não foi feita reposição de tentativas incorretas).

\subsubsection{Análise de erros}

Apesar do número de erros em uma tarefa de tempo de reação a estímulos supra limiares como os que usamos nesse trabalho ser relativamente baixo, fizemos uma análise utilizando a discriminabilidade dos estímulos. A ideia por trás dessa análise é que a discriminabilidade dos estímulos nas tarefas utilizadas aqui, em que o indivíduo precisa identificar o estímulo antes de selecionar a resposta, pode ser alterada pela velocidade com que o indivíduo inicia o movimento após o aparecimento do estímulo: dependendo da estratégia adotada, o indivíduo poderia liberar a inibição motora proativa antes de a identificação do estímulo ter sido completada. 
Para as respostas certas e erradas foram calculadas a taxa de hits (número de tentativas em que a pessoa respondeu para um alvo e acertou, dividido pelo número de tentativas com esse alvo) e a taxa de alarme falso (número de tentativas em que a pessoa respondeu para um alvo e errou, dividido pelo número total de tentativas com o outro alvo). A partir dessas taxas foi calculada a medida de discriminabilidade (d' prime), dada pela fórmula Z(hits) - Z(alarmes falsos), onde Z é a inversa da função de densidade cumulativa da distribuição normal padrão.

\subsection{Medidas Eletroencefalográficas (EEG)}

O uso do eletroencefalograma permite a medida contínua da atividade do cérebro de maneira não invasiva, em tempo real, com uma resolução temporal da ordem de 1ms. Essa característica torna o EEG uma ferramenta muito adequada para estudos de cronometria mental, registrando processos não observáveis em medidas de tempo de reação (Luck, 2014).

Acredita-se que os potenciais elétricos observados com as medidas do EEG reflitam a atividade pós-sináptica de populações de neurônios das células piramidais do córtex (Woodman, 2010). Os potenciais registrados têm uma relação sinal-ruído muito baixa, por isso os registros são filtrados e processados de acordo com os objetivos de um experimento.

É possível utilizar um marcador para o aparecimento de um estímulo ao longo de todo o experimento, de modo que, posteriormente, se possa fazer a média do registro em um intervalo de tempo relacionado ao aparecimento do(s) estímulo(s) de interesse (potencial relacionado a eventos - PRE). A partir dessa média, observa-se a presença diferentes componentes - que podem ser definidos, de maneira superficial, como deflecções positivas e negativas de voltagens com uma determinada distribuição no escalpo, que aparecem em uma determinada sequência e apresentam características razoavelmente constantes (como amplitude e latência) entre indivíduos saudáveis da mesma faixa etária quando estes são expostos a condições equivalentes. Por exemplo, após o aparecimento de um estímulo visual a que um indivíduo deve responder apertando um botão, podem seguirse os componentes P1, N1... (Luck, 2014).

A partir de elementos de um componente, como a amplitude, a latência, a distribuição no escalpo e diferenças nas suas características de acordo com as condições de 
um experimento, costuma-se inferir os processamentos cerebrais e os processos cognitivos a que estão associados. No entanto, não é possível definir com exatidão a fonte dos sinais de EEG observados no escalpo devido a uma dificuldade conhecida como problema inverso: matematicamente, há infinitas fontes que poderiam dar origem aos sinais observados (Woodman, 2010). Apesar disso, com o uso de um grande número de eletrodos durante o registro e a aplicação de métodos sofisticados de modelagem, em conjunto com outras medidas como imagens por ressonância magnética estrutural e funcional, é possível fazer uma estimativa bastante razoável acerca das estruturas geradoras de componentes específicos (Di Russo et al., 2001).

Neste trabalho vamos abordar os componentes P1, N1 e P3 associados ao aparecimento de uma pista visual periférica e ao aparecimento de um estímulo alvo que os indivíduos deveriam identificar e a que deveriam responder apertando um botão com o dedo indicador da mão esquerda ou da mão direita de acordo com a identidade do alvo.

O componente P1 é comumente observado com amplitude máxima em eletrodos occipitais laterais. Ele pode ser dividido em duas fases: uma fase inicial, com latência média entre 72 e 80 ms, amplitude máxima entre 98 e 110 ms, contralateral ao lado de aparecimento do estímulo visual e origem estimada no córtex dorsal extra estriado do giro occipital medial; e uma fase tardia, com latência média entre 110 e 120 ms, amplitude máxima entre 136 e 146 ms, ipsilateral ao lado de aparecimento do estímulo visual e origem estimada no córtex ventral extra estriado do giro fusiforme (Di Russo et al., 2001). A fase inicial do componente P1 pode estar associada à ativação das áreas V3N3a e de áreas adjacentes do giro occipital medial; a fase tardia do componente P1 pode estar associada à ativação da porção anterior da área estendida de V4v do giro fusiforme (Di Russo et al., 2001). O componente P1 é sensível aos parâmetros dos estímulos e pode ser modulado pelo processamento atencional e pelo estado de alerta do indivíduo (Luck, 2014).

O complexo de componentes negativos observados após o aparecimento do componente P1 é formado por quatro subcomponentes, e os que nos interessam aqui são o primeiro, denominado N150, observado em eletrodos occipito-parietais no hemisfério contralateral ao aparecimento do estímulo visual, e o quarto subcomponente, denominado N200, observado em eletrodos occipto-parietais no hemisfério ipsilateral ao aparecimento do estímulo visual, que aqui chamamos de N1 (Di Russo et al., 2001). 
O componente P3 é observado em diversas condições experimentais, e apresenta diferentes distribuições no escalpo de acordo com essas condições, mas não existe um consenso quanto aos processos cognitivos e neurofisiológicos envolvidos no aparecimento desse componente. O processamento mais citado referente ao P3 é definido de uma maneira geral como um processo de atualização da representação do contexto ambiental induzida pelo aparecimento de um estímulo, e pode estar relacionado à elaboração de uma estratégia, uma vez que pode aparecer muito tarde para influenciar a resposta. Além disso, a amplitude do componente P3 parece ser menor quando o indivíduo tem alguma incerteza quanto a um estímulo ser ou não o alvo e quando os estímulos são apresentados em um curto intervalo de tempo; a latência é proporcional ao tempo necessário para categorizar o estímulo; e sugere-se que esteja associado a um processo de inibição neural (Polich, 2007; Luck, 2014).

De acordo com a topografia e as condições experimentais utilizadas, o componente P3 pode ser classificado como P3a e P3b. O componente P3b é máximo em regiões parietais, e está associado ao processamento de um estímulo alvo. Sugere-se que tenha origem em atividades na região temporais e parietais, que recebe densas conexões norepinefrinérgicas (Polich, 2007).

O componente P3a tem amplitude máxima em eletrodos centro-parietais e tem amplitude diminuída quando um estímulo é apresentado repetidamente, estando possivelmente associado à resposta de orientação. Com uma topografia mais centralizada, define-se uma variação do componente P3a, o P3 "não-vai", associado a processos de inibição de uma resposta quando é apresentado um estímulo distraidor e a tarefa envolve uma discriminação mais fácil. Sugere-se que esteja relacionado mecanismos frontais de focalização atencional e memória mediados por atividade dopaminérgica (Polich, 2007). 


\section{CONCLUSÃO}

Os indivíduos são capazes de utilizar regularidades estatísticas complexas do ambiente como pista para alocação atencional e de aprender essas regularidades de maneira implícita, após poucas repetições.

Estratégias de controle motor inibitório, adotadas a partir da representação de eventos em um dado contexto, interagem com os efeitos atencionais observados em tarefas de tempo de reação. 


\section{REFERÊNCIAS*}

Albares M, Criaud M, Wardak C, Nquyen SC, Ben Hamed S, Boulingquez P. Attention to baseline: does orienting visuospatial attention really facilitate target detection? J Neurophysiol. 2011; 106(2):809-16.

Anderson B. There is no such thing as attention. Front. Psychol. 2011; 2:246.

Angelucci A, Bullier J. Reaching beyond the classical receptive field of V1 neurons: horizontal or feedback axons? J Phys. 2003; 141-54.

Ashby FG, Maddox WT. Human category learning. Annu Rev Psychol. 2005; 56:149-78.

Awh E, Belopolsky AV, Theeuwes J. Top-down versus bottom-up attentional control: a failed theoretical dichotomy. Trends Cogn Sci. 2012; 16(8): 437-43.

Balota DA, Yap MJ. Moving beyond the mean in studies of mental chronometry: The power of response time distributional analysis. Ass Psychol Sci. 2011; 20(3): 160-66.

Braver TS. The variable nature of cognitive control: a dual mechanism framework. Trends Cogn Sci. 2012; 16:106-13.

Boulinguez P, Ballanger B, Granjon L, Benraiss A. The paradoxical effect of warning on reaction time: demonstrating proactive response inhibition with event-related potentials. Clin Neurophysiol. 2009; 120(4):730-7.

Boulinguez $\mathrm{P}$, Jaffard $\mathrm{M}$, Granjon L, Benraiss A. Warning signals induce automatic EMG activations and proactive volitional inhibition: evidence from analysis of error distribution in simples RT. J Neurophysiol. 2008; 99(3):1572-8.

Burle B, Vidal F, Tandonnet C, Hasbroucq T. Physiological evidence for response inhibition in choice reaction time tasks. Brain Cogn. 2004; 56(2): 153-64.

Burnham BR. Displaywide visual features associated with a search display's appearance can mediate attentional capture. Psychon Bull Rev. 2007; 14(3):392-422.

Burnham BR, Neely JH. A static color discontinuity can capture attention when a target is an abrupt-onset singleton. J Exp Psychol Hum Percetpt Perform. 2008; 34(4):831-41.

Cai W, Oldenkamp CL, Aron AR. A proactive mechanism for selective suppression of response tendencies. J Neurosc. 2011; 31(16): 5965-9.

\footnotetext{
* De acordo com: International Committee of Medical Journal Editors. [Internet]. Uniform requirements for manuscripts submitted to biomedical journals [2011 Jul 15] Available from: http://www.nlm.nih.gov/bsd/uniform_requirements.html.
} 
Carmel T, Lamy D. Towards a resolution of the attentional-capture debate. J Exp Psychol Hum Percept Perform. 2015; 41(6)1772-82.

Carrasco M, Yeshurum Y. Covert attention effects on spatial resolution. Prog Brain Res. 2009; 176:65-86.

Carreiro LRR, Haddad Jr H, Baldo MVC. The modulation of simple reaction time by the spatial probability of a visual stimulus. Braz J Med Biol Res. 2003; 36(7):907-11.

Charles L, Van Opstal F, Marti S, Dehaene S. Distinct brain mechanisms for conscious versus subliminal error detection. Neuroimage. 2013; 73:80-94.

Ciaramitaro VM, Cameron EL, Glimcher PW. Stimulus probability directs spatial attention: an enhancement of sensitivity in humans and monkeys. Vis Res. 2001; 41:57-75.

Criaud M, Wardak C, Hamed SB, Ballanger B, Boulinguez P. Proactive inhibitory control of response as the default state of executive control. Front Psychol. 2012; 3:59.

Di Russo F, Martinez A, Sereno MI, Pitzalis S, Hillyard AS. Cortical Sources of the early componentes of the visual evoked potential. Hum Brain Map. 2001; 15:95-111.

Eimer M, Kiss M. Involuntary attentional capture is determined by task set: Evidence from event-related brain potentials. J Cong Neurosc. 2008; 20(8):1423-33.

Eimer M, Kiss M. Top-down search strategies determine attentional capture in visual search: Behavioral and electrophysiological evidence. Atten Percept Psychophys. 2010; 72(4): 95162.

Eimer M, Kiss M, Press C, Sauter D. The roles of feature-specific task set and bottom-up salience in attentional capture: an ERP study. J Exp Psychol Hum Percep Perform. 2009; 35(5):1316-28.

Favre E, Ballanger B, Thobois S, Broussolle E, Boulinguez P. Deep brain stimulation of the subthalamic nucleus, but not dopaminergic medication, improves preactive inhibitory control of movement initiation in Parkinson's disease. Neurotherapeutics. 2013; 10(1):15467.

Feher da Silva C, Baldo MV. A simple artificial life model explains irrational behaviour in human decision-making. Plos One. 2012; 7(5):e34371.

Feher da Silva C, Baldo MV. Computational models of the Posner simple and choice reaction time tasks. Front Comput Neurosci. 2015; 9:81.

Feher da Silva C, Victornio CG, Caticha N, Baldo MVC. Exploration and recency as the main proximate causes of probability matching: a reinforcement learning analysis. bioRxiv. 2017; 104752. 
Folk CL, Remington RW, Johnston JC. Involuntary covert orienting is contingent on attentional control settings. J Exp Psychol Hum Percept Perform. 1992; 18(4): 1030-44.

Folk $\mathrm{CL}$, Remington RW, Wright JH. The structure of attentional control: contingent attentional capture by apparent motion, abrupt onset, and color. J Exp Psychol Hum Percept Perform. 1994; 20(2): 317-29.

Folk CL, Remington RW, Wu SC. Additivity of abrupt onsets effects supports nonspatial distraction, not the capture of spatial attention. Atten Percept Psychophys. 2009; 71(2): 30813.

Fuga NB. The influence of attention and forward masking on reaction time. [Dissertação (Mestrado em Fisiologia Humana)]. São Paulo: Instituto de Ciências Biomédicas, Universidade de São Paulo; 2002.

Gaissmaier W, Schooler $\amalg$. The smart potential behind probability matching. Cognition. 2008; 109(3):416-22.

Gonçalves PMR. How the placeholders on the screen influence the manifestation of automatic attentional effect. [Dissertação (Mestrado em Neurosciência)]. São Paulo: Instituto de Psicologia, Universidade de São Paulo; 2015.

Goschy H, Bakos S, Müller HJ, Zehetleitner M. Probability cueing of distractor locations: both intertrial facilitation and statistical learning mediate interference reduction. Front Psychol. 2014; 5:1195-

Guedes VA. Inhibitory effects of a visual cue in reaction time tasks. [Dissertação (Doutorado em Fisiologia Humana)]. São Paulo: Instituto de Ciências Biomédicas, Universidade de São Paulo; 2007.

He X, Fan S, Zhou K, Chen L. Cue validity and object-based attention. J Cogn Neurosci. 2004; 16(6):1085-97.

Hopf JM, Luck SJ, Girelli M, Hagner T, Mangun GR, Scheich H, Heinze HJ. Neural sources of focused attention in visual search. Cereb Cortex. 2000; 10(12):1233-41.

Huettel SA, Mack PB, McCarthy G. Perceiving patterns in random series: dynamic processing of sequence in prefrontal cortex. Nat Neurosci. 2002; 5(5):485-90.

James W. The principles of psychology. New York: Cosimo, Inc; 2007. v.1, 712 p.

Jaffard M, Benraiss A, Longcamp M, Velay JL, Bounlinguez P. Cueing method biases in visual detection studies. Brain Res. 2007; 1179: 106-18.

Jaffard M, Longcamp M, Velay JL, Roth $M$, Nazarian B, Boulinguez P. Proactive inhibitory control of movement assessed by event-related fMRI. Neuroimage. 2008; 42(3):1196-206. 
Jahanshahi M, Obeso I, Rothwell JC, Obesa JA. A fronto-striato-subthalamic-palidal network for goal-directed and habitual inhibition. Nat Rev Neurosci. 2015; 16(12):719-32.

Jonides J. Towards a model of the mind's eye's movement. Canad J Psychol. 1980; 34(2):10312.

Jonides J. Further toward a model of the mind's eye's movement. Bull Psychon Soc. 1983; 21(4):247-50.

Kabata T, Matsumoto E. Cueing effects of target location probability and repetition. Vision Res. 2012; 73:23-9.

Kafaligönül H, Breitmeyer BG, Ögmen H. Effects of contrast polarity in paracontrast maskint. Atten Percept Psychophy. 2009; \&1(7):1576-87.

Kaitz M, Monitz J, Nesher R. Electrophysiological correlates of visual masking. Intern J Neurosci. 1985; 28:261-68.

Keysers C, Perret DI. Visual masking and RSVP reveal neural competition. Trends in Cogn Sci. 2002; 6(3):120-25.

Kiss M, Van Velzen J, Eimer M. The N2pc component and its links to attention shifts and spatially selective visual processing. Psychophysiology. 2008; 45(2):240-49.

Klein, RM. Inhibitory tagging system facilitates visual search. Nature. 1988; 334: 430-1.

Klein, RM. Inhibition of return. Trends in Cogn Sci. 2000; 4(4):138-47.

Kopp B, Seer C, Lange F, Kluytmans A, Kolossa A, Fingscheidt T, Hoijtink H. P300 amplitude variations, prior probabilities, and likelihoods: A Bayesian ERP study. Cogn Affect Behav Neurosci. 2016; 16:911-28.

Krauzlis RJ, Bollimunta A, Arcizet F, Wang L. Attention as na effect not a cause. Trends Cogn Sci. 2014; 18(9):457-64.

Kuhn TS. A estrutura das revoluções científicas. São Paulo: Perspectiva; 2011. 260 p.

Luce RD. Response times: Their role in inferring elementary mental organization. New York: Oxford University Press; 1986. 579 p.

Luck SJ. An introduction to the event-related potential technique. $2^{\text {nd }}$ ed. Massachusetts: MIT Press; 2014. 575 p.

Macaluso E, Doricchi F. Attention and predictions: control of spatial attention beyond the endogenous-exogenous dichotomy. Front Hum Neurosch. 2013; 7:685. 
Macknik SL, Livingstone MS. Neuronal correlates of visibility and invisibility in the primate visual system. Nat Neurosc. 1998; 1(2):144-49.

Macknik SL, Martinez-Conde S, Haglund MM. The role of spationtemporal edges in visibility and visual masking. Proc Nat Acad Sci Unit Stat Am. 2000; 97(13):7556-60.

Mazza V, Turatto M, Caramazza A. Attention selection, distractor suppression and N2pc. Cortex. 2009; 45(7): 879-90.

Meyer HC, Bucci DJ. Neural and behavioural mechanisms of proactive and reactive inhibition. Learn Mem. 2016; 23(10):504-14.

Miller J. A warning about median reaction time. J Exp Psychol Hum Percept Perform. 1998; 14(3):539-43.

Mlodinow, L. O andar do bêbado: como o acaso determina nossas vidas. Rio de Janeiro: Zahar, 2011. $322 \mathrm{p}$.

Moore T, Armstrong KM, Fallah M. Visuomotor origins of covert spatial attention. Neuron. 2003; 40(4):671-83.

Näätänen R, Gaillard AW, Mäntysalo S. Brain potential correlates of voluntary and involuntary attention. Prog Brain Res. 1980; 54:343-8.

Olton, DS, Schlosberg, P. Food-searching strategies in young rats: Win-shift predominates over win-stay. J Comp Physiol Psychol. 1978; 92(4):609-18.

Osborne J. Notes on the use of data transformations. Pract Assessm Res Eval. 2002; 8(6):1-7.

Oskarsson AT, Van Boven L, McClelland GH, Hastie R. What's next? Judging sequences of binary events. Psychol Bull. 2009; 135(2):262-85.

Otten M, Schreij D, Los SA. The interplay of goal-driven and stimulus-driven influences on spatial orienting. Atten Percept Psychophys. 2016; 78:1642-54.

Otto AR, Taylor EG, Markman AB. There are at least two kinds of probability matching: Evidence from a secondary task. Cognition. 2011; 118:274-79.

Peirce JW. PsychoPy - Psychophysics software in Python. J Neurosck Methods. 2007; 162(12):8-13.

Peirce JW (2009) Generating stimuli for neuroscience using PsychoPy. Front. Neuroinform. 2009; 2:10.

Pfefferbaum A, Ford JM, Weller BJ, Kopell BS. ERPs to response production and inhibition. Electroenceplahogr Clin Neurophysiol. 1985; 60(5):423-34. 
Polich J. Updating P300: An integrative theory of P3a and P3b. Clin Neurophysiol. 2007; 118(10):2128-48.

Posner MI. Timing the brain: Mental chronometry as a tool in neuroscience. PloS Biol. 2005; 3(2):e51.

Posner MI, Cohen Y. Components of visual orienting. Attention and Performance: Control of language processes. 1984. 531-56.

Prinzmetal W, McCool C, Park S. Attention: reaction time and accuracy reveal different mechanisms. J Exp Psychol Gen. 2005; 134(1): 73-92.

Ratcliff R. Methods for dealing with reaction time outliers. Psychol Bull. 1993; 114(3):510-32.

Ratcliff R, McKoon G. The diffusion decision model: theory and data for two-choice decision tasks. Neural Comput. 2008; 20(4):873-922.

Reber AS. Implicit learning and tacit knowledge. J Exp Psychol Gen. 1989; 118(3):219-35.

Remington RW, Folk CL, McLean JP. Contingent attentional capture or delayed allocation of attention? Percept Psychophys. 2001; 63(2):298-307.

Romberg AR, Saffran JR. Statistical learning and language acquisition. Wiley Interdiscip Rev Cogn Sci. 2010; 1(16):906-14.

Sais FA, Ribeiro-do-Valle LE. Absence of placeholders abolishes exogenous attentional effect in a peripheral cueing procedure. Intern J of Brain and Cogn Sci. 2013; 2(1):1-8.

Schapiro A, Turk-Browne N. Statistical learning. Brain Mapping: An Encyclopedic Reference. 2015. Vol. 3, pp 501-6.

Shepherd M, Findlay JM, Hockey RJ. The relationship between eye movements and spatial attention. Q J Exp Psychol A. 1986; 38(3):475-91.

Smith JL, Johnstone SJ, Barry RJ. Movement-related potentials in the Go/NoGo task: the P3 reflects both cognitive and motor inhibition. Clin Neurophysiol. 2008; 119(3):704-14.

Sperling G, Dosher BA. Strategy and optimization in human information processing. In Boff K, Kaufman L, Thomas J (Eds), Handbook of Perception and Performance. Vol. 1. New York: Wiley. Chapter 2, pp1-65.

Stuphorn V, Emeric EE. Proactive and reactive control by the medial frontal cortex. Front. Neuroeng. 2012; 5:9.

Stuphorn V. Neural mechanisms of response inhibition. Curr Op Behav Sci. 2015; 1:64-71. 
Stemmler M, Usher M, Niebur E. Lateral interactions in primary visual cortex: a model bridging physiology and psychophysics. Science. 1995; 269(5232):1877-80.

Theeuwes J. Top-down and bottom-up control of visual selection. Acta Psychol (Amst). 2010; 135(2):77-99.

Tom SM, Fox CR, Trepel C, Poldrack RA. The neural basis of loss aversion in decision-making under risk. Science. 2007; 315(5811):515-8.

van der Haijden AHC. Probability matching in visual selective attention. Can J Psychol. 1989; 43(1):45-52.

van der Meer $M$, Kurth-Nelson Z, Redish AD. Information processing in decision-making systems. Neuroscientist. 2012; 18(4):342-59.

van Gaal S, Lamme VA. Unconscious high-level information processing: implication for neurobiological theories of consciousness. Neuroscientist. 2012; 18(3):287-301.

Victorino CG. Estudo do desenvolvimento de estratégias decisionais em escolhas binárias repetidas. [Dissertação (Mestrado em Fisiologia Humana)]. São Paulo: Instituto de Ciências Biomédicas, 2012.

Vossels S, Thiel CM, Fink GR. Cue validity modulates de neural correlates of covert endogenous orienting of attention in parietal and frontal cortex. Neuroimage. 2006; 32(3):1257-64.

Wardak C, Ramanoël S, Guipponi O, Boulinguez P, Ben Hamed S. Proactive inhibitory control varies with task context. Eur J Neurosc. 2012; 36(11): 3568-79.

Whelan R. Effective analysis of reaction time data. Psychol Rec. 2008; 58:475-82.

Woodman GF. A brief introduction to the use of event-related potentials (ERPs) in studies of perception and attention. Atten Percept Psychophys. 2010; 72(8):2031-46.

Woodman GF, Arita JT, Luck SJ. A cuing study of the N2pc componente: An index of attentional deployment to objects rather than spatial locations. Brain Res. 2009; 101-11.

Yin HZ, Li D, Yang J, Li W, Qiu J, Chen YY. Neural bases of unconscious error detection in a Chinese anagram solution task: Evidende from ERP study. PoS One. 2016; 11(5):e0154379.

Yantis S. Stimulus-driven attentional capture and attentional control settings. J Exp Psychol Hum Percept Perform. 1993; 19(3):676-81. 